\title{
La solidaridad en familias atípicas
}

\author{
Graciela Sarrible \\ Universitat de Barcelona. Facultat de Ciències Econòmiques. Departament de Sociologia. \\ Avinguda de la Diagonal, 690. 08034 Barcelona. Spain
}

\section{Resumen}

La solidaridad no constituye un objeto de estudio frecuente en sociologia y menos aún en el grupo familiar. A través de unas entreviscas y de un análisis cualitativo, se analizan las opciones, las motivaciones y los sentimientos que rodean a las personas que ayudan a los demás.

Se trata de situaciones especiales y de soluciones particulares. Estos casos no responden a la media ni intentan abarcar todo el panorama de posíbilidades. Al contrarto, se trata de considerar opciones "dímites" para entender los aspectos que intervienen en las decisiones tomadas en el grupo familiar.

Los géneros tienen comportamientos diferentes, sobre todo respecto a la reclusión o a la participación en otras actividades. El punto de vista de las trabajadoras que se dedican a cuidar enfermos está contemplado y comparado con los familiares que realizan la misma tarea. Las contradicciones que incurten durante la entrevista permite vislumbrar sentimientos opuestos que no desmerecen el valor de la tatea realizada.

Palabras clave: solidaridad, familias atípicas, cuidados, enfermos.

\section{Abstract. Solidarity in non common families}

Solidarity is not a commen subject of study in Sociology, not even in the research of the family. This work is based in a qualicative analysis of the interviews. Feelings, behavours and reasons of the persons who takes care of the others are considered.

Only particular situations are studied. They are not commons, not mediums. They are boundary possibilities so as to understand the background of the decisions.

Gender behave distinnctivly, not only in the family but also in relation of outside activities. The point of view of workers is also considered in comparison with not-workers. The contradictions let us perceive opposites feelings, that don't devalue the task.

Key words: solidaricy, nom common families, care, ill.

\section{Sumario}

Introducción Discusión

Arálisis Bibliografía 


\section{Introducción}

La tesis de los investigadores anglosajones que la familia es un eje central para la comprensión de la cultura contemporánea puede ser aplicada al mundo mediterráneo. Esta idea, desarrollada por el constructivismo social, permite centrar ciertos análisis del cambio social alrededor del núcleo familiar, de la vida privada y de los valores que dan prioridad a las relaciones afectivas respecto a las cuestiones materiales, como trabajo, éxito o dinero (Gubrim y Holstein, 1990).

El terreno de los sentimientos no ha estado abonado en sociología. Desde $\mathrm{M}$. Weber se lo considera como un componente de la acción no racional. Las corrientes posteriores, sobre todo las que centran sus teorías en la elección racional, no incluyen estos aspectos como decisorios. El poder de la norma resulea una explicación sociológica, mientras que las ciencias de la conducta proveen las explicaciones sobre los sentimientos, a partir de situaciones individuales.

En la última década, cuando el modelo más estable y difundido de familia desde la Revolución Francesa ha comenzado a perder su hegemonfa frente a otros alternativos, la reconsideración de ciertos aspectos hasta ahora postergados como los sentimientos, forma parte de la crítica de los viejos modelos. Sin embargo, todavia no ha pasado a integrar nuevas teorias o respuestas.

Existe una cierta apertura para considerar los sentimientos como un elemento a tener en cuenta en el estudio de la familia: si el modelo anterior de pareja se podía basar en la norma, la inestabilidad presente puede tener como componente esencial los sentimientos mutuos de satisfacción-insatisfacción que manifiestan sus integrantes.

El estudio de la solidaridad, como uno de los sentimientos de grupos primarios, se ha desarrollado en las ciencias sociales, sobre todo en la historia social y en la antropología. La documentación sobre asociaciones de ayuda mutua ha permitido reconstruir manifestaciones de solidaridad concretas y grupales (Universiré de Nice, Solidarités, affinités et groupementes sociaux dans les pays mediterranéens, Nice, 1982).

Sin embargo, mucha menos atención se ha prestado al terreno de la solidaridad familiar. Si dejamos de lado los discursos morales que hacían de la familia el centro de un universo de buenos sentimientos, no han sido numerosas las aportaciones en este terreno. La razón principal es la anteriormente apuntada: la solidaridad, como sentimiento, no era considerada un objeto de estudio sociológico, al menos digno de mayor crédito.

En la medida en que en una encuesta se plantea la cuestión de la solidari$\mathrm{dad}$, la respuesta inmediata se refiere a las personas cercanas. Se ha demostrado que se trata, en general, de personas del ámbito familiar, más que de amigos. En primer lugar, se solicita la ayuda de los padres, sobre todo en el caso de parejas jóvenes, y, sólo después, del resto de parientes (Bonvalet y otros, 1993).

En las familias rucleares (pareja e hijos), los abuelos son los primeros a quienes se dirige la solicitud; tanto cuando se trata de ayuda económica como 
de cuidar a los niños pequeños o resolver problemas cotidianos. La cuestión que puede resultar inhabitual es cómo resolver dificultades o situaciones excepcionales, tanto en el caso de familias nucleares, como en otras de composición diversa de la media. Esta cuestión resultó central en la investigación'!

Cuando los núcleos que tienen miembros dependientes (niños o ancianos) no pueden (o no quieren) ayudar en circunstancias inusuales, como hospitalizaciones prolongadas o enfermedades crónicas; entonces la cuestión es quién puede encargarse de ellas. El espíritu de solidaridad familiar es el que da respuestas a estas situaciones. En ausencia de un miembro de la familia, habrá otro disponible. Sin embargo, no siempre se alcanza una situación satisfactoria para todas las partes.

Se han realizado veintiséis entrevistas en Cataluña para estudiar las respuestas de los diferentes miembros de una familia en momentos críticos. Las entrevistas se han circunscrito a personas que por razones familiares o como trabajo se encargaban de otras en situación de dependencia. El objetivo de esta investigación es el de replantear el estudio de los sentimientos como un aspecto que tendría que ver con las relaciones familiares, en tanto relaciones sociales. Incorporar esta dimensión, hasta ahora casi postergada, implica resaltar todos los aspectos contradictorios que contiene, tanto en el ámbito del discurso como en la justificación de la acción. El objetivo más empírico reposa en el análisis de los mecanismos de toma de decisión en situaciones críticas.

La metodología cualitativa ha sido escogida en la medida en que se la ha considerado un instrumento adecuado para una primera aproximación a cuestiones internas de la familia, a sentimientos y motivaciones, que son sujeto de difícil medición. Se ha estimado que esta opción era válida para poder relacionar las opiniones y razones expuestas como justificación de los comportamientos.

No se trat 6 , en ningún caso, de presentar todo el abanico de posibilidades y soluciones que concurren en casos críticos. Se ha tratado de recoger los sentimientos y las motivaciones de las personas que deciden, libremente o no, hacerse cargo de los demás. Conocer el marco en el que se toman estas decisiones, las alternativas que se evalúan y la opinión sobre su labor constituían cuestiones que se intentan dilucidar.

La razón por la cual se ha considerado a las familias escogidas en la encuesta como "atípicas" reside en que su estructura no corresponde a las categorias más extendidas. Todas las personas interrogadas viven en familia, si consideramos la acepción menos restringida del término ${ }^{2}$. Una abuela que se encarga de sus nietos constituiría una familia. Un hijo adulto que cuida su madre octogena-

1. Fsra investigación fue financiada con fondos del Ministerio de Educación y Ciencia (SEC920460).

2. Sobre la acepción del término familia: A propósito de la definición del concepto de familia, Graciela Sarrible, DT9401. Documents de Treball. Facultat de Ciències Econòmiques i Empresarials. Universitat de Barcelona, 1994. 
ria, con la cual ha vuelto a vivir luego de la disolución de un matrimonio, constituye la persona principal de la familia y del hogar. Una mujer empleada para ocuparse de una persona mayor que vive en su hogar mientras cría a su hijo durante ocho años, constituye un caso límite de la definición de familia. Se estima, entonces, que viven en familia. Pero no se tendrán en cuenta exclusivamente las relaciones categorizables que se establecen entre ellas, sino también los sentimientos que se generan.

La limitación que implica la elección de las entrevistas significa que las respuestas constituyen puntos de vista posibles, no necesariamente únicos. Se trata sólo de una visión, entre otras,. No se intenta abarcar todas las posibilidades. Avanzar explicaciones tentativas que constituyeran opciones satisfactorias por sí solas o complementarias de otras es el objetivo de este trabajo.

Los principales temas que han surgido de este análisis, y que se exponen a continuación, son tres. En primer lugar, los comportamientos y actitudes de hombres y mujeres difieren. Delinear características por géneros permite insistir en una constante disparidad, aún cuando se trate de desempeñar un mismo papel dentro del ámbito familiar. En segundo lugar, las personas que ocupan este lugar pero no pertenecen a la familia denotan sentimientos y motivaciones similares a los anteriores. En tercer lugar, las oposiciones entre los sentimientos expresados a través de la entrevista son motivo de un análisis particular. Las contradicciones, constantes en estas encuestas, sirven para desvelar la oposición entre la obligación moral, los afectos y las exigencias de esta tarea. La discusión final recoge las interpretaciones propuestas en el marco de una generalización que sirve de conclusión al trabajo.

\section{Análisis}

Existen rasgos comunes entre la mayoría de las historias de vidas recogidas. En primer lugar, existen numerosos casos en que los familiares han cambiado temporalmente de domicilio para poder cuidar a alguien. El segundo aspecto a destacar es que, en general, no se trata de la primera vez que ocurre. La misma persona que ahora se muestra solidaria, en el pasado ya ha ayudado a otros familiares en situaciones semejantes. En la misma familia, las historias se repiten con un mismo personaje.

Se puede suponer que algunas personas están siempre disponibles o se ofrecen voluntarias, mientras que otras no. Se diría que sienten una cierta obligación de encargarse de cuidar a los demás. Este marco moral se muestra en oposición con los servicios profesionales (casas de reposo, asilos, cuidadoras profesionales) que se encargan de personas enfermas. Cuando el miembro de la familia se ofrece o se considera necesario es porque está persuadido que los profesionales prestan un servicio insuficiente o caro. La ternura y la compañia tendrían más importancia que la atención especializada.

Si bien se trata de una elección, no es libre. Las personas que ayudan manifiestan sentimientos contradictorios hacia las que están a su cargo, de condena hacia los que no colaboran o no actúan como ellas, pero sobre todo respecto 
de su propia situación. Declaran que actúan por amor, pero no pueden evitar que el entorno que se supone que han escogido les deprima. La contradicción también se manifiesta entre el ofrecimiento aparentemente libre y sus propias declaraciones de no encontrar otro que los reemplace.

Los sentimientos expresados deben ser interpretados en el contexto de la entrevista en que cada uno justifica sus propios méritos. El resto de familiares, que no tienen un comportamiento "tan solidario o desinteresado", según los propios entrevistados, son mal considerados y convertidos en culpables de los males que han sucedido o podrían todavía acontecer. La voluntariedad del ofrecimiento no resulta como libre o primera opción, sino como el resultado de la eliminación del resto de posibilidades.

Los temas expuestos a continuación han permitido plantear una serie de interrogantes. Estas cuestiones constituyen el nudo de las interpretaciones que surgen del análisis. Ellas son:

1. Autosuficiencia. La familia expresa sentimientos de autosuficiencia. Si falta un miembro, debe haber otro que pueda reemplazarlo. Las personas manifiestan una cierta obligación moral. La cuestión consiste en dilucidar hasta qué punto la voluntariedad en la prestación de servicios puede constituir el eje de la elección.

2. Confusión de sentimientos. Los sentimientos de solidaridad se confunden en muchos casos con la claridad. La negociación, entendida como un trato entre iguales en el seno de la familia, no resulta evidente cuando se trata de personas dependientes. El respeto por la opinión o el deseo de los otros no siempre forma parte de la toma de decisiones. Es importante saber si las personas ancianas expresan libremente su opción y si ésta se toma en consideración.

3. La exclusiva responsabilidad. Los miembros de la familia que se encargan del cuidado de otros se sienten como los únicos responsables. No ven alternativas a su labor y a su dedicación, aún cuando en la familia otros miembros han realizado la misma labor en el pasado, en situaciones semejantes. El desprecio a los demás forma parte del sentimiento que los legitima en su papel.

4. Contradicciones. Muchos de los sentimientos expresados resultan contradictorios. La obligación se enfrenta a una cierta libertad de elección verbalizada en el discurso. Se ama a la persona que se cuida, pero no se la soporta. No existe aceptación o rechazo, sino una amalgama de sentimientos contrapuestos.

Cuando se trata de cuidados, no por azar se piensa en la mujer. Si en la mayoría de los casos es un familiar femenino y cercano el que realiza la tarea, es válido considerarlo el término de comparación con los otros casos: trabajadoras y hombres. Si la mujer realiza un trabajo no remunerado y reconocido que sólo le otorga poder de decisión, la trabajadora representa la cara inversa. 
En el caso del hombre, sus expectativas también son diferentes. Se mueve con más independencia, no sufre frecuentes depresiones y logra el reconocimiento de su tarea al ocuparse de los demás de forma organizada. Sin embargo, los tres protagonistas mencionados se mueven en un mar de contradicciones. Sus deseos de reconocimiento contrastan con una imagen social negativa y sus valoraciones de otros miembros de la familia no tienen en cuenta experiencias pasadas. El desarrollo de este análisis valorará estos aspectos.

\section{Los géneros}

La frecuentación de la intensidad de las relaciones familiares en los países mediterráneos se presenta, según los resultados de las encuestas, como más elevada que en otras zonas próximas (De Miguel, 1992). Entre los jóvenes casados, la necesidad de visitar a la madre parece más fuerte que la de ver al padre. La figura femenina constituye todavia un punto de apoyo y referencia para los jóvenes. En España, como en Italia, los jóvenes no manifiestan ninguna urgencia o necesidad de dejar el hogar de sus padres para vivir solos o en pareja (Vallés, 1992).

Esta diferencia de trato se debe a que las expectativas respecto al padre y a la madre resultan distintas. Se espera de cada uno un tipo de ayuda particular. No se le pide lo mismo. Mientras que se acude al padre en busca de apoyo material, a la madre se le demandan servicios, además de quizás un mayor apoyo afectivo.

No se trata de que a los enfermos o a los niños los cuiden sólo las mujeres, pero es lo que frecuentemente ocurre. Parece como si los hombres sintieran la obligación o se plantearan hacerlo sólo cuando no hubiera mujeres disponibles. Sin embargo, esta labor genera respuestas diferenciadas por parte de cada uno de los sexos.

Los resultados de la encuesta muestran que es la madre la que decide de ocuparse de los jóvenes adultos cuando están enfermos. Es la madre la que toma las decisiones de traslado del hogar para mejorar la calidad de vida o estar más cerca de los centros asistenciales. El papel del padre, como bien describen muchos teóricos de la familia, no queda bien perfilado (Roussel, 1989). El padre parece aceptar las decisiones que se toman más que participar activamente en ellas cuando la situación es crítica, y será la madre la que preste la mayor dedicación de su tiempo en el futuro.

La mujer toma las decisiones directamente cuando se trata de sus propios padres y tiene voz y voto en el caso de que tenga que ocuparse de sus suegros. Aún cuando los hijos tienen la última palabra, al ser las nueras la que permanecen al lado de los enfermos y que ofrecen su tiempo, esta situación condiciona las opciones y la decisión final que toman sus maridos.

La persona que se encarga de los enfermos se convierte en la persona principal. Ello significa que no es solamente la responsable, sino también aquella que sustenta la autoridad o tiene un voto de calidad. Lamentablemente, los enfermos son rara vez escuchados. Se debería tener en cuenta su opinión en 
la medida en que se está tomando una decisión respecto a su vida. En cambio, constituye a lo sumo un elemento más, sin revestir habitualmente la consideración de fundamental.

En vez de la madre, otra mujer puede reemplazarla y adquiere la importancia del papel que ejerce. En los casos de enfermos de sida, si no existía una madre próxima, otra mujer se convertía en la compañera y aún la esposa de la persona enferma. En estos casos, las mujeres expresaban pena y no una obligación moral ancestral de ayuda. Aún cuando estuvieran obnubiladas por sentimientos de fatalidad, estas mujeres expresaban su deseo de ayudar y acompañar al enfermo. Son conscientes que sin su ayuda la persona que quieren se sentirá infeliz o desprotegida. En esa medida, su actitud puede estar condicionada por esa necesidad. Dicen no sentirse obligadas a ocuparse ellas mismas, pero la pregunta de quién puede reemplazarla queda sin respuesta.

La figura de la buena samaritana podrá ser revalorizada en este contexto. Ni contenta, ni triste. Es el sentido común o la caridad cristiana interiorizados en la infancia lo que las moriva en sus acciones. Estas personas se sienten más responsables que obligadas. Este sentimiento predomina frente al rechazo o a las depresiones que comporta ser restigos del deterioro de un familiar próximo. No abandonarían al enfermo. Una cierta añoranza de la líbertad perdida, que esperan recuperar más tarde, contribuye a vivir esta situación exclusivamente como transitoria.

Cuando no hay mujeres, son los hombres los que asumen la tarea. En estos casos, el hombre no se considera que está adoptando un papel femenino, sino que es su obligación atender a su madre o a su hijo. Los hombres resultan ser más duros que las mujeres en sus juicios condenatorios acerca de los demás miembros de la familia que no ofrecen su ayuda. Igual que en el sexo femenino, se sienten las únicas personas capaces de cumplir con esta tarea. Los otros hermanos son despreciados, explf́citamente o a través de un discurso indirecto, por la falta de colaboración que se visualiza tan libre como egoísta.

Cuando se trata de una mujer, no las tratan de igual manera. Se diría que los hombres resultan menos exigentes con el otro sexo, de la misma manera que las mujeres son más duras con sus congéneres que con el sexo masculino. Estos hombres critican duramente a sus hermanos, pero se muestran más comprensivos en sus juicios acerca de sus ex-mujeres, por ejemplo.

En los dos casos de hombres entrevistados, ambos se encontraban en el paro. La principal razón esgrimida fue que no podían continuar trabajando y ocupándose de su madre hospitalizada. Pero la diferencia sustancial respecto a las mujeres se basa en que contribuyen con su trabajo y su tiempo en asociaciones voluntarias. No sólo piensan en su propio caso, en la atención de sus respectivas madres, sino también en las restantes personas que se encuentran en similar situación. Por ello despliegan una actividad voluntaria y organizada para que otros también puedan contar con una cierta ayuda.

Mientras que las mujeres se enfrentan con su tarea, entendida como personal y privada, los hombres generalizan su problema intentando encontrar soluciones para los desconocidos. Si las mujeres permanecen casi recluidas en 
su hogar, los hombres sienten la necesidad de salir. Pensar en los demás constituye la justificación de sus escapadas y de las ausencias del hogar. Dispuestos a pagar a otra persona para que los reemplace, el hecho de desarrollar otras actividades les permite reencontrar la estima perdida por la falta de trabajo retribuido.

Se ha mencionado a la familia, a los próximos, a los parientes. Hasta estos momentos parecía como si la ayuda se prestara exclusivamente entre aquellos que tenían una relación consanguínea. Puede ser cierto para la mayoría de los casos, pero estas tareas no siempre están realizadas por personas con lazos de sangre.

\section{Las trabajadoras}

Las mujeres que cuidan a personas dependientes como trabajo retribuido expresan sentimientos particulares y distintivos de otros empleos. Se sienten parte integrante de la familia. Según sus propias declaraciones, no aceptarían mejores ofertas para continuar ocupándose de las personas que están a su cargo. Manifiestan sentimientos de ternura y amor hacia las personas que cuidan que pretenden correspondidos.

Igual que los miembros de la familia que se desempeñan en las mismas tareas, las trabajadoras consideran que ocuparse de personas enfermas o ancianas constituye una obligación. Sin embargo, reconocen que su presencia tiene un cariz complementario. En la medida en que la vida moderna exige nuevas formas de organización del tiempo, admiran o desprecian a las familias a partir de la dedicación y atención que prestan a la persona que cuidan, independientemente de su permanente y constante presencia.

También comparten con los familiares que tienen la misma ocupación el sentimiento de ser irreemplazables. Suponen equivocadamente que la persona podria morir si la abandonan y cambian de empleo. Se sienten tan cercanas y responsables de la persona que cuidan como los miembros de la familia que desempeñan la misma tarea. El sentimiento más contradictorio con su posición es el desprecio que manifiestan por la contrapartida en dinero que reciben por su trabajo, explicando que su permanencia obedece a los buenos sentimientos que las animan.

Resulta cuando menos sorprendente la convergencia de sentimientos y justificaciones de personas tan diversas. No era de esperar que todas las personas manifestaran homólogos sentimientos y motivaciones. Una cierta generosidad, como también la necesidad de reconocimiento por los sentimientos que pretenden compartidos, constituyen la llave de su permanencia y de su falta de reconocimiento de alternativas posibles.

Se esgrime el amor como la razón prioritaria. Un amor que fue recibido en el seno de la familia de origen y que se puede devolver en estos momentos. En cambio, la obligación moral constituye la respuesta intempesriva e inmediata. Los sentimientos de amor y ternura compensarían la tristeza y la falta de libertad. La obligación representaría la razón de permanencia que otorga la fuerza necesaria para continuar. 
Al inicio de la convivencia en el hogar de las personas que deben cuidar, las trabajadoras pasan por un periodo de adaptación dificil. Esta situación se prolonga hasta el día en que pueden controlar - pero también soportar- las exigencias del enfermo. Es sólo en ese momento que deciden quedarse.

Si no se llegara a resolver satisfactoriamente la convivencia, entonces se manifiestan disponibles para cualquier cambio de empleo, por ende de hogar. Las excusas presentadas a la ocasión son de distinto cariz, pero en general se refieren a la falta de colaboración de la familia del enfermo, a su descuido y a la falta de interés que demuestran. De igual manera que cuando sienten que no existe un apoyo de parte de la familia, prefieren partir.

No solamente necesitan cierto reconocimiento de su labor por parte de las personas que las contratan, también esperan de ellas qute mantengan su participación en las decisiones que deben ser tomadas. Prefieren compartir la responsabilidad que de otro modo podría resultarles excesiva. Cuentan con que los familiares puedan acudir en caso de crisis. No son ellas las que desean tomar las decisiones dificiles.

Las etapas en la adaptación de la relación enfermo-cuidadora implican la continua consolidación de su posición interna en el hogar y con la familia. Soportar el mal humor del enfermo tiene como contrapartida la aceptación de su situación y de su posición, en primer lugar, por parte del enfermo y, en segundo lugar, por parte de los demás.

El inicio de la relación puede resultar conflictivo si los enfermos no fueron consultados o no escogieron esta solución. De ahí que el rechazo sea frecuente. Ellas mismas manifiestan que el enfermo esperaba que la propia familia se ocupara y no una persona ajena a ella. Ello significa que una de las formas de lograr la aceptación pasa por convencer ai enfermo de que la situación en que se encuentra, si no es la ideal, al menos es la mejor solución a la cual pueden optar aquellas personas que le aman.

En una segunda etapa, las personas enfermas se resignan a la permanencia de un extraño, pero consideran que cualquier otro sería mejor. En realidad, la aceptación no es tal: se disfraza de un cambio de persona y no de situación, que parece asumida. Ésta será una de las últimas reivindicaciones que puedan ser planteadas con total libertad de criterio y elección. En estos casos, la alternativa que se le presenta al enfermo es la de internación en un asilo o aceptación total de la persona y de la situación en que se encuentra. Todo se resuelve con el abandono de la lucha y las reivindicaciones por un cambio de parte del enfermo.

Las trabajadoras, por su parte, manifiestan la necesidad de ser amadas. Desean valorar su trabajo a través del reconocimiento de sentimientos que pretenden sean recíprocos. No solamente se exige del enfermo esta relación afectiva, sino también a los familiares próximos, sobre todo a aquellos que la han contratado. Resulta evidente que no quieren ser tratadas como asalariadas. Esperan dignificar su tarea a través de la valorización que los demás hacen de ella. Las expresiones verbales de reconocimiento resultan, en general, suficientes a este efecto. 
La integración a las familias se lograría por esta vía. Dejar de ser consideradas empleadas, ser tratadas en tanto miembro del grupo, revalorizar la tarea realizada constituyen los medios de adaptación e incorporación. Cuando no logran esta situación, la familia del enfermo es despreciada. Se estima que lo abandonan, que no lo visitan con frecuencia. En realidad, se están buscando las oportunidades en que la integración pueda producirse, en que el reconocimiento por el trabajo sea constante y en que el acercamiento sea permanente.

Esta necesidad de integración puede explicarse también por la razón de que muchas de ellas son extranjeras. A falta de otros empleos a los que puedan optar, las agencias se nutren de personas recién llegadas. La teoría de la red del migrante también sirve para explicar la concentración en determinadas áreas o sectores de la gente que proviene de un mismo origen.

Una trabajadora dominicana expresaba que las migrantes latinoamericanas se encargan de estas tareas porque las españolas no desean realizarlas. Por otra parte, un familliar de un enfermo sostenf́a lo mismo. Quizás sea una visión simplificada de la realidad. Los trabajos menos valorados (que no necesican una formación técnica específica) y por ende mal retribuidos, son ocupados en Europa hace largo tiempo por los trabajadores inmigrados.

Cuidar enfermos o personas de edad avanzada constituye una de las actividades socialmente poco valoradas. Ello explicaría porqué las trabajadoras que ocupan estos puestos buscan un reconocimiento en su entorno inmediato. Los sentimientos compartidos y la integración reemplazan la imagen social negativa. De puertas para adentro, la realidad tiene que ser otra.

La situación de la trabajadora puede ser definida por una serie de aspectos que la caracterizan y que se suceden en el tiempo desde el momento inicial. En primer lugar, el deseo de sentimientos compartidos puede ser una forma de superar el rechazo inicial que los enfermos han hecho ostensible. En segundo lugar, quieren ser valoradas positivamente por el entorno inmediato, o sea, la familia. En tercer lugar, prefieren negar su condición de trabajadoras para integrarse como miembro en el hogar. En cuarto y último lugar, pueden desestimar otras ofertas de trabajo en la medida en que juzgan por los sentimientos y no por el salario que reciben o les ofrecen fuera.

En este ámbito, si bien la mayor parte de las trabajadoras provenían de agencias que se mantenian en contacto con ellas; existe también trabajo no declarado. Por lo visto, hay dos alternativas corrientes para encontrar este tipo de trabajo. Por una parte, están las empresas; por la otra, las redes sociales. Cuando algunas personas necesitan un ingreso extraordinario, buscan en el vecindario familias que necesiten un apoyo para cuidar un enfermo.

\section{Las contradicciones}

En todos los casos no resulta difícil encontrar sentimientos contrapuestos. Existe un terreno privado que se oculta al entrevistador. Los sentimientos se expresan de manera diferente en el transcurso de la entrevista. Las personas se 
muestran por momentos nerviosas, en otros tristes o preocupadas. Resultaría sospechoso que en un terreno donde se manifiestan los afectos, el entrevistado se mostrara impasible. A esta limitación, propia del tema tratado, se suma el hecho de que muchas de las personas que debían ser cuidadas presentaban enfermedades crónicas irreversibles, sobre todo neurodegenerativas, que contribuían a un estado general de desaliento.

En estas circunstancias, los familiares reconocen que estas personas, tan encantadoras y amables en otro tiempo, se vuelven tiránicas y exigentes. Estas manifestaciones corresponden frecuentemente a hijas que se encargan de sus madres. La explicación que esgrimen se basa en que existiría una vuelta atrás con la edad: los ancianos tendrían las exigencias de los niños. Se desea acaparar la atención del entorno a través de peticiones que son consideradas como caprichos.

Las personas podrían manifestar un comportamiento entendido como egoísta en la medida en que se sienten inútiles. Si no tienen ocupación, pedirían a los demás que ocupen su tiempo con ellas. Sin embargo, si se recuperan los roles tradicionales, las personas ancianas podrían sentirse revalorizadas ayudando ellas a los demás, de la misma manera que los otros también les prestan su ayuda. Una situación más igualitaria o de cierta reciprocidad podría contribuir a que las obligaciones puedan ser sentidas por ambas partes, que la ayuda que se presta fuera recíproca.

La obligación moral constituye la razón esgrimida por la mayoría de las personas que se encargan de los enfermos. Sin embargo, no es más que la última alternativa cuando todas las demás fueron descartadas. Durante la lectura de las entrevistas se comprende que las personas que permanecen prestando cuidados lo hacen por haber rechazado las otras opciones sustitutivas. En la escala de valores, la obligación moral está por encima de todas las demás en la jerarquía.

Los sentimientos de ser irreemplazables son compartidos tanto por los familiares como por las trabajadoras. Este sentimiento implica, por una parte, una culpabilización de lo que podría ocurrir de no permanecer. Por la otra, el resto de la familia es menospreciada en la medida en que no presta suficiente ayuda. La autoestima se acrecienta en la medida en que se entroniza la tarea que se realiza y se desprecia a los demás por no demostrar la misma generosidad.

Estas personas encargadas de cuidar a otras declaran que los otros se han negado a ayudar. En muchos casos declaran que no se ha planteado ni siquiera la solicitud. No resulta evidente, a partir de la reconstrucción de las historias, que los restantes familiares sean tan indiferentes o egoístas como el entrevistado desea presentarlos. Otras veces, la imagen negativa que se han construido de los demás resulta incoherente con otros aspectos del relato.

El poder de estas personas reside en su autoafirmación como irreemplazables. No solicitan ayuda o la techazan por no considerarla suficientemente desinteresada. Esto permite sobreponerse a la tristeza y depresión que puede generar el contacto permanente con enfermos, crónicos o terminales. En la 
medida en que no existen otras posibilidades, deben tener la fuerza de continuar. Sin embargo, las depresiones sobrevienen o se manifiestan cuando las personas a las que cuidan mueren.

\section{Discusión}

La solidaridad familiar se desarrolla a partir de una red de parientes próximos. Sin embargo, los miembros de una farnilia no constituyen una comunidad igualitaria, con los mismos derechos y obligaciones. Es la mujer sobre todo la que siente la necesidad de ocuparse de las personas que necesitan ayudas y cuidados. Esta exigencia de su tiempo y de su disponibilidad la hace merecedora de un poder de decisión que no tendría en otra circunstancia. Los hombres aparecen como meros sustitutos en su ausencia.

Se debe de tener en cuenta que antes del deterioro, las personas mayores convivieron generalmente en pareja, tuvieron hijos que partieron y luego sobrevino la muerte del cónyuge. Pudieron haber permanecido solas muchos años en la medida en que manifestaban su autonomía e independencia de los demás. En ocasiones, permanecieron internadas en centros colectivos, generalmente privados, hasta encontrar una solución más satisfactoria para los miembros de la familia. En estos casos, la última elección se basa sobre todo en los sentimientos que expresa tanto el enfermo, que no desea terminar su vida fuera del hogar donde ha vivido, como en los sentimientos, posiblemente de culpabilidad, de las personas responsables de la decisión de su internamiento.

Las historias que se han analizado no dejan de responder a un esquema de valores tradicionales, de solidaridad familiar y convivencia de varias generaciones en su mismo hogar. Si se evalúa la historia de las familias, se puede considerar que, en el pasado, la solidaridad demostrada en esta disponibilidad para ayudar a los otros era más frecuente que en la actualidad. Evidentemente, la Guerra Civil en España alteró la vida familiar. Muchos ejemplos de ayuda en esos momentos aparecieron en los relatos recogidos. Las personas de más de cincuenta años encuestados han padecido, de alguna manera, las consecuencias de ese conflicto.

En todas las familias existen muchos ejemplos de casos de solidaridad sin contrapartida. Pero esa parte de la historia corresponde sobre todo al mundo rural, a la pequeña aldea o a una convivencia próxima y estrecha. En ese mundo resultaba más fácil cambiar de domicilio para prestar ayuda, aún cuando el tiempo de permanencia no estuviera acotado al principio.

Todas las personas que hoy se preocupan por los demás habían tenido múltiples y buenos ejemplos en el pasado. Muchas de ellas habían sido criadas por sus abuelos o convivieron durante un tiempo con otros familiares. En otros casos, familias monoparentales debido a la muerte de uno de los cónyuges, han recibido la ayuda de los otros.

Las familias en el pasado podían ser autosuficientes. La proximidad de los diferentes domicilios y el trabajo en un mismo sector podrían favorecer los 
intercambios, en general de carácter temporal. Si la muerte era más frecuente, las largas convalecencias por enfermedades crónicas eran excepcionales.

Las migraciones separaron a los parientes. A pesar de ello, en todos los casos analizados existía más de un miembro de la familia que pudiera, eventualmente, hacerse cargo de las personas enfermas. Lo que puede resultar paradójico es que sea frecuentemente la misma persona la que va a cuidar a sucesivos enfermos.

Todos reconocen que esta persona se ofrece voluntaria, lo que resulta conveniente para los demás. Siempre resulta más fácil cuando hay alguien que se brinda a hacerlo. Ello no implica que exista una sola persona disponible en grupos amplios, sino que se aprovecha la buena voluntad y la experiencia para que cumpla con la misma tarea en sucesivas ocasiones.

No descartan las familias la utilización de servicios externos: las residencias y trabajadores fijos en el domicilio. En el caso de las personas encuestadas, la imagen de los hogares colectivos resulta deplorable. Cuando se refieten a terceras personas que han sido internadas por sus familiares, les hacen culpables en una asociación estrecha de abandono y muerte. Por ello, las residencias han sido utilizadas como recurso temporal, hasta una nueva organización del tiempo de los familiares o hasta la contratación de una persona permanente en el hogar. El paso por estas residencias sirve de preparación para la aceptación de un extraño en el hogar. La vuelta al hogar se recibe a cambio de la nueva situación. Las opciones del enfermo en este caso resultan limitadas. Tiene que elegir entre dos opciones no satisfactorias: o acepta a la persona de fuera impuesta o volverá a la residencia.

Las trabajadoras que se ocupan de estas tareas no se consideran como tales. Aparentemente, según lo manifestado, los sentimientos y la valorización de su tarea les permitiría desestimar las reglas del mercado laboral. Ellas juegan a ser buenas samaritanas. No se ven a sí mismas como trabajadoras, puesto que no juzgan las oportunidades por el nivel de salario que les ofrecen o por las condiciones de otro empleo. La fidelidad al enfermo que cuidan y a la familia tiene como contrapartida el ser reconocida como parte integrante y no como una extraña.

No queda claro el punto de vista de los enfermos. En realidad, la percepción de necesidades y adaptación de los enfermos se obtiene de terceras personas, puesto que en la mayoría de los casos ya habían fallecido. Se conoce sus manifestaciones porque los otros miembros de la familia son capaces de reconocer la disparidad de criterios que los enfrentaron en ciertos momentos y a propósito de sus propias condiciones de vida.

Es justo reconocer que existen grandes dosis de solidaridad real y desinteresada en los casos recogidos. Ello no quita que la generosidad pueda aparecer mezclada con cierta obligación moral o culpabilización de no actuar como se espera de uno. Estos sentimientos encontrados hacen aflorar las dudas sobre las intenciones manifestadas o sobre la certeza de ciertas devociones.

Las motivaciones de las personas que se ocupan de otros resultan bastante complejas. El amor y la ternura constituyen la primera justificación de la tarea 
que se lleva a cabo. La segunda sería la reciprocidad. Se ha recibido en otros momentos y ahora es el momento en que se debe retribuir. La solidaridad en el ámbito familiar resulta un complejo mundo del cual se tiene pocos indicios hasta el presente. Pero la integración de los sentimientos en el marco explicativo de la dinámica de la familia como grupo se hace necesaria.

\section{Bibliografía}

Bonvaler, Catherine y otros (1993). "Proches et Parents". Population, 48, 1, enerofebrero. París: INED, p. 83-110.

DAvICO, R. (1982). "Solidarité, affinités et groupements sociaux en milieu urbain: spectographie d'une ville, Turin dans les années 1980\%. En Actes des Journées d'Etudes: Solidarités, affinités et groupements sociaux dans les pays méditerranéens (xvie-xxe siècles). Cahiers de la Méditerranée. Niza: Centre de la Mediterranée Moderne et Contemporaine, p. 25-47.

DE Migel, Amando (1992). La sociedad española: 1992-1993. Madrid: Alianza.

DEL CAMPO, Salustiano (1991). La nueva familia española. Madrid: EUDEMA, Actualidad.

Gubrim, Jaber F. \& Fames, A. Holstein (1990). What is family? California, USA: Mayfield.

Oruzo, Francisco Andrés (1991). Los nuevos valores de los españoles. Madrid: Fundación Santa María.

Roussel, Louis (1989). La familie incertaine. París: Odile Lacob.

SARRible, Graciela (1994). A propósito de la definición del concepto de familia. DT, 9401. Documents de Treball. Facultar de Ciències Econòmiques i Empresarials. Batcelona: Universitat de Barcelona.

VALlEs, Miguel S. "Los jóvenes y la constitución de los nuevos hogares y famitias". En A. De Miguel, op. cit, p. 151-222.

VovelLe, M. (1982) «Dix ans de sociabilité méridionalem. En Actes des Jornées d'Etudes: Solidarités, affinités et groupements sociaux dans les pays méditerranéens (xvie-xxe siècles). Cahiers de la Méditerranée. Niza: Centre de la Méditerranée Moderne et Contemporaine, p. 149-157. 